\title{
COMPUESTOS BIOACTIVOS Y ANÁLISIS SENSORIAL DE UNA BEBIDA FUNCIONAL DE MAÍZ MORADO (Zea mays L.) Y ESTEVIA (Stevia SP.)
}

\author{
Bioactive compounds and sensorial analysis of functional drink from purple corn (Zea mays L.) \\ and stevia (Stevia sp.)
}

Pablo E. Bonilla ${ }^{1}$, Fredy Quispe ${ }^{3}$, Luisa Negrón ${ }^{2}$, Amparo I. Zavaleta $^{2}$

${ }^{1}$ Instituto de Investigación de Ciencias Farmacéuticas y Recursos Naturales "Juan de Dios Guevara”; ${ }^{2}$ Instituto de Investigación de Química Biológica, Microbiología y Biotecnología "Marco Antonio Garrido Malo”; ${ }^{3}$ Centro Latinoamericano de Enseñanza e Investigación en Bacteriología Alimentaria - CLEIBA. Facultad de Farmacia y Bioquímica. Universidad Nacional Mayor de San Marcos.

\section{RESUMEN}

En la presente investigación se evaluaron los compuestos bioactivos, y se determinaron la actividad antioxidante y el grado de satisfacción de una bebida de maíz morado y estevia, mediante los métodos por HPLC, ABTS, ORAC, DPPH y por comparación según la escala hedónica. Los resultados mostraron compuestos fenólicos cercanos a 2 mg/mL, altos contenidos de antocianinas (50,84 mg/100 mL) y actividad antioxidante según ABTS (17,96 $\mu \mathrm{mol}$ TEAC/mL), ORAC (31,01 $\mu \mathrm{mol} \mathrm{TEAC} / \mathrm{mL})$ y DPPH (o,706 GAE $\mathrm{mg} / \mathrm{mL})$. La evaluación de vida útil reveló un efecto sinérgico entre las antocianinas del maíz morado y los componentes activos de la estevia. El grado de satisfacción reveló que el formulado se califica entre "me gusta mucho" (19\%) y "me gusta ligeramente" (33\%) según escala hedónica sobre los atributos de color, olor, sabor y aceptabilidad en general.

Palabras clave: Maíz morado, Zea mays, estevia, antocianinas, ABTS, ORAC, DPPH.

\section{SUMMARY}

In the present study were evaluated the bioactive compounds and antioxidant activity and satisfaction degree of a drink made with purple corn and stevia. Were used HPLC, ABTS, ORAC, DPPH methods and was done comparison according to the hedonic scale. Results showed close phenolic compounds to $2 \mathrm{mg} / \mathrm{mL}$, high in anthocyanins (50,84 mg/10o $\mathrm{mL})$ and antioxidant activity with ABTS (TEAC 17,96 pmol/mL), ORAC (31,01 $\mu \mathrm{mol}$ TEAC/mL) and DPPH (GAE o,706 mg/mL). The useful life assessment revealed a synergistic effect between the purple corn anthocyanins and the active components of stevia. The satisfaction degree revealed that the formulation was described between "really like " (19\%) and "like slightly " (33\%) according to the hedonic scale about attributes of color, smell, taste and overall acceptability.

Keywords: Purple corn, Zea mays, stevia, anthocyanins, ABTS, ORAC, DPPH.

\section{INTRODUCCIÓN}

a dislipidemia constituye uno de los factores de riesgo más importantes en el desarrollo de las enfermedades cardiovasculares (ECV) que desencadenan principalmente en infarto de miocardio o accidente cerebrovascular ${ }^{(1,2)}$. Hacia el 2030, se estima que a nivel mundial morirán cerca de 23,3 millones de personas por ECV, sobre todo por cardiopatías y accidente cerebrovascular (ACV) y si no se cambian los hábitos alimentarios y estilo de vida se prevé que seguirán siendo la principal causa de muerte en el mundo ${ }^{(3)}$. En el Perú, el 2008 se registraron cerca de 19\% de muertes producidas por ECV. Según estudio realizado en ciudades de América Latina, los factores de riesgo metabólico con mayor prevalencia son sobrepeso, hipercolesterolemia e hipertensión arterial ${ }^{(4)}$. En ese sentido, el consumo de alimentos funcionales se presenta como una alternativa para prevenir los riesgos deenfermedades cardiovasculares. Entre estos alimentos se encuentra el maíz morado, de reconocida actividad antioxidante, hipolipemiante, antiinflamatoria e hipotensora, según estudios realizados en modelos experimentales en pacientes ${ }^{(5)} \mathrm{y}$ animales de experimentación ${ }^{(6-10)}$, y estevia que contiene edulcorantes no calóricos que sustituyen a la sacarosa y fructosa, con propiedades cardioprotectoras, hipoglicemiantes y antiateroescleróticas ${ }^{(11)}$. A nivel mundial, el consumo de estos alimentos se incrementa día a día y con el objetivo de aprovechar recursos de la biodiversidad peruana, se evaluaron sus componentes bioactivos, actividad antioxidante y el grado de satisfacción de una bebida en base a maíz morado y estevia. 
Tabla 1. Compuestos fenólicos presentes en el formulado de maíz morado y estevia.

\begin{tabular}{|c|c|c|c|}
\hline № & $\underset{(\min )}{\operatorname{Tr}}$ & $\begin{array}{l}\text { Concentración } \\
\text { (mg/100 mL)* }\end{array}$ & Compuesto fenólico asignado \\
\hline 1 & 21,86 & 1,48 & Ácido protocatéquico \\
\hline 2 & 25,88 & 0,35 & Derivado de ácido cinámico ${ }^{\mathrm{a}}$ \\
\hline 3 & 36,25 & 0,41 & Derivado de ácido cinámico ${ }^{a}$ \\
\hline 4 & 39,28 & 0,03 & Ácido clorogénico \\
\hline 5 & 40,28 & 0,13 & Derivado de ácido cinámico ${ }^{\mathrm{a}}$ \\
\hline 6 & 43,61 & 0,00 & Derivado de flavona $^{\mathrm{d}}$ \\
\hline 7 & 44,63 & 6,42 & Derivado de Antocianina $^{e}$ \\
\hline 8 & 47,79 & 136,17 & $\begin{array}{l}\text { Desconocido (cuantificado } \\
\text { como ácido protocatéquico) }\end{array}$ \\
\hline 9 & 48,39 & 2,40 & Derivado de Antocianina $^{e}$ \\
\hline 10 & 50,11 & 14,18 & Derivado de Antocianina $^{\mathrm{e}}$ \\
\hline 11 & 53,02 & 0,48 & Ácido orto-cumárico \\
\hline 12 & 53,56 & 26,40 & Derivado de Antocianina $^{e}$ \\
\hline 13 & 55,38 & 0,22 & Derivado de ácido cinámico ${ }^{\mathrm{a}}$ \\
\hline 14 & 56,14 & 0,95 & Derivado de Antocianina $^{\mathrm{e}}$ \\
\hline 15 & 56,76 & 0,49 & Derivado de Antocianina $^{e}$ \\
\hline 16 & 59,16 & 0,35 & Derivado de flavonol $^{\mathrm{b}}$ \\
\hline 17 & 59,54 & 0,46 & Derivado de flavonol $^{\mathrm{b}}$ \\
\hline 18 & 61,37 & 0,99 & Derivado de flavonol $^{\mathrm{b}}$ \\
\hline 19 & 61,63 & 0,94 & Derivado de ácido cinámico ${ }^{a}$ \\
\hline 20 & 63,03 & 0,16 & Derivado de flavonol $^{\mathrm{b}}$ \\
\hline 21 & 63,24 & 0,98 & Derivado de flavonol $^{\mathrm{b}}$ \\
\hline 22 & 63,91 & 2,29 & Derivado de ácido cinámico ${ }^{\mathrm{a}}$ \\
\hline 24 & 65,74 & 0,56 & Derivado de flavona $^{c}$ \\
\hline 25 & 66,27 & 1,99 & Derivado de ácido cinámico ${ }^{\mathrm{a}}$ \\
\hline 26 & 83,52 & 0,20 & Derivado de flavonol $^{\mathrm{b}}$ \\
\hline 27 & 87,52 & 0,30 & Derivado de ácido cinámico ${ }^{a}$ \\
\hline \multicolumn{2}{|c|}{ Total } & 200,09 & \\
\hline
\end{tabular}

* Promedio de dos repeticiones. $\mathrm{Tr}=$ Tiempo de retención en minutos. ${ }^{\mathrm{C} C}$ Cuantificado como ferúlico a $320 \mathrm{~nm}$, buantificado como quercetina a $360 \mathrm{~nm}$, 'Cuantificado como luteolina a $320 \mathrm{~nm},{ }^{\mathrm{d}}$ Cuantificado como apigenina a $320 \mathrm{~nm},{ }^{\mathrm{e}} \mathrm{C}$ antificado como cianidina-3-glucósido a $520 \mathrm{~nm}$.

\section{MATERIAL Y MÉTODOS}

\section{Lugar de ejecución}

Planta Piloto de Alimentos Funcionales de la Facultad de Farmacia y Bioquímica de la Universidad Nacional Mayor de San Marcos.

\section{Materiales y reactivos}

El material vegetal constituido por mazorcas de maíz morado se colectó de enero a marzo de 2013 en el distrito de Omas, provincia de Yauyos, departamento de Lima.

Todos los reactivos fueron de grado analítico.

\section{Formulación de la bebida}

En planta piloto se preparó el formulado de maíz morado y estevia, según el diagrama de flujo de la figura 1. Se utilizó ácido ascórbico y clavo de

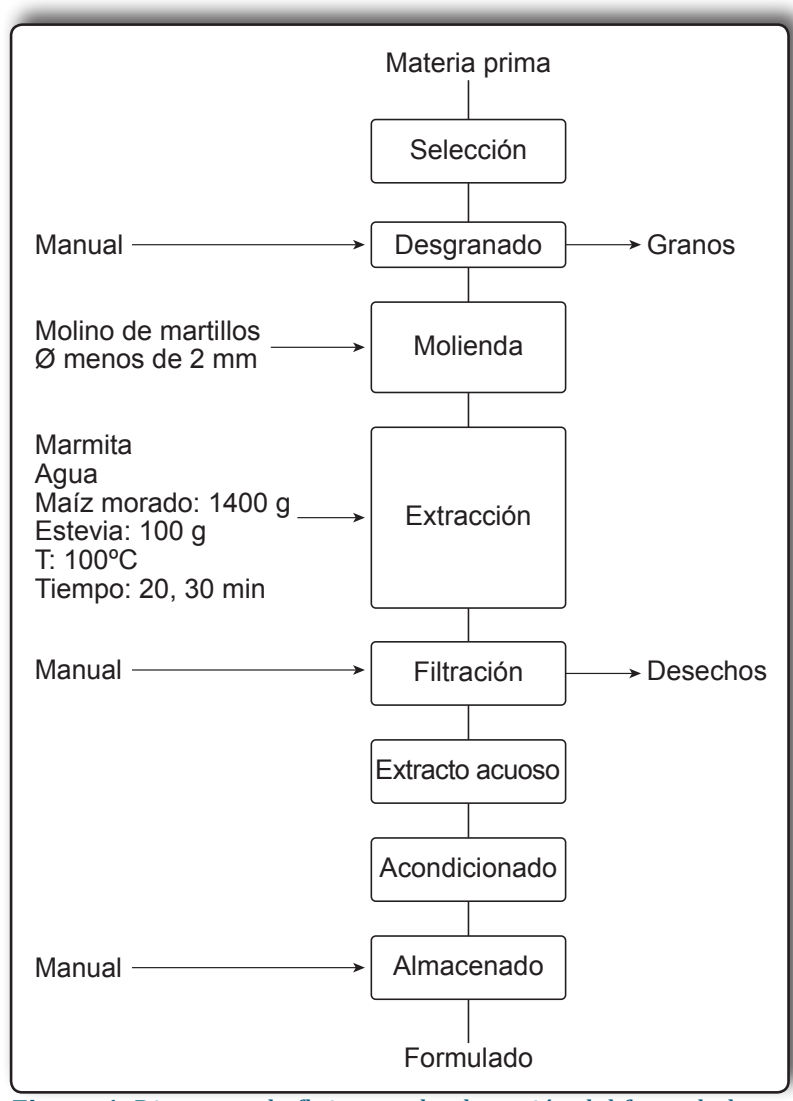

Figura 1. Diagrama de flujo para la obtención del formulado.

olor para acondicionar el formulado y mejorar su aceptabilidad.

\section{Determinación de antocianinas según el método de $\mathrm{pH}$ diferencial}

El formulado de maíz moradoy estevia, se filtró sobre papel Whatman $\mathrm{N}^{\circ} 1$ y los filtrados se hicieron pasar por membranas de filtración de $0,45 \mu \mathrm{m}$ para la determinación de antocianinas, según el método de $\mathrm{pH}$ diferencial, utilizando el espectrofotómetro UV-Vis (Spectroquant PHARO 300) y su contenido se expresó como cianidina-3-glucósido ${ }^{(12)}$.

\section{Determinación de fenoles totales}

Los fenoles totales de las muestras se evaluaron según el método modificado de Folin-Ciocalteu a 765 $\eta \mathrm{m}$ en el espectrofotómetro UV-Vis y su contenido se expresó como mg de equivalentes de ácido gálico (GAE) por g de muestra ${ }^{(13,14)}$.

\section{Determinación de la actividad antioxidante según DPPH}

El efecto antioxidante del formulado sobre el radical estable 2,2-difenil-1-picrilhidracilo (DPPH) se 


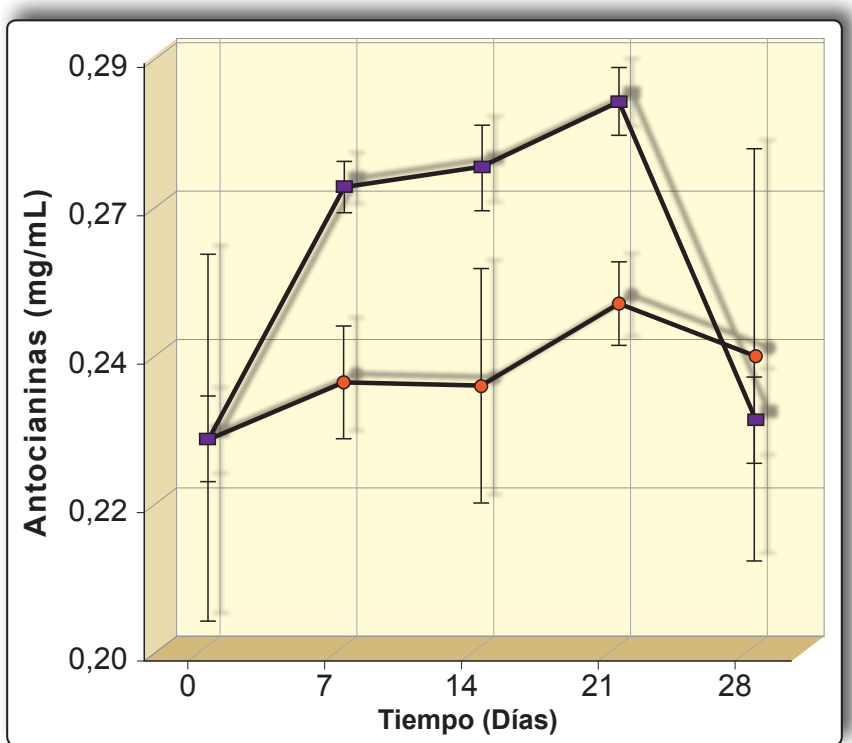

Figura 2. Antocianinas en los formulados de maíz morado y estevia bajo condiciones de temperatura ambiente "๑" y refrigeración “口”. Valores promedio de 3 repeticiones con su respectiva desviación estándar.

evaluó a 517 ๆm en el espectrofotómetro UV-Vis, y la actividad antioxidante se reportó como porcentaje de inhibición de DPPH (\%).

\section{Determinación de actividad antioxidante según ABTS y ORAC}

La actividad antioxidante del formulado se evaluó según los métodos ABTS y ORAC, los resultados se expresaron como $\mu$ mol de equivalentes Trolox $(\text { TEAC })^{(15,16)}$.

\section{Evaluación de compuestos fenólicos por HPLC-PAD}

El análisis de compuestos fenólicos en el formulado se evaluó sobre un módulo de separación Waters 2695 (Waters, Milford, MA) equipado con un auto inyector de columna HPLC de fase reversa, un detector de arreglo de fotodiodos 996 (PAD) y el software Empower. Los datos espectrales se registraron desde 200 a 700 ๆm durante una corrida total. Una columna X-terra RP-18 $(5 \mu, 250$ $\mathrm{x}$ 4,6 mm) de Waters y un guarda columna de 4,6 x 2,0 $\mathrm{mm}$ fueron usados para la separación de fenólicos a $30^{\circ} \mathrm{C}$. La fase móvil estuvo compuesta por el solvente (A) agua : ácido fórmico (95:5, v/v, $\mathrm{pH} 2$ ) y el solvente (B) acetonitrilo. El gradiente de solventes fue: $\mathrm{O}-15 \% \mathrm{~B}$ en 40 $\min , 15-45 \%$ B en 45 min, y 45-100\% B en 10 min. Se usó una velocidad de flujo de $0,5 \mathrm{~min} / \mathrm{mL}$ y se inyectaron 20 $\mu \mathrm{L}$ de muestra. El formulado y la fase móvil se filtraron a través de un filtro Millipore de $0,22 \mu \mathrm{m}$, tipo GV (Millipore, Bedford, MA) previo a la inyección al HPLC. Los compuestos fenólicos se identificaron y cuantificaron por sus tiempos de retención y espectros UV-Vis de estándares previamente inyectados al equipo ${ }^{(17)}$.

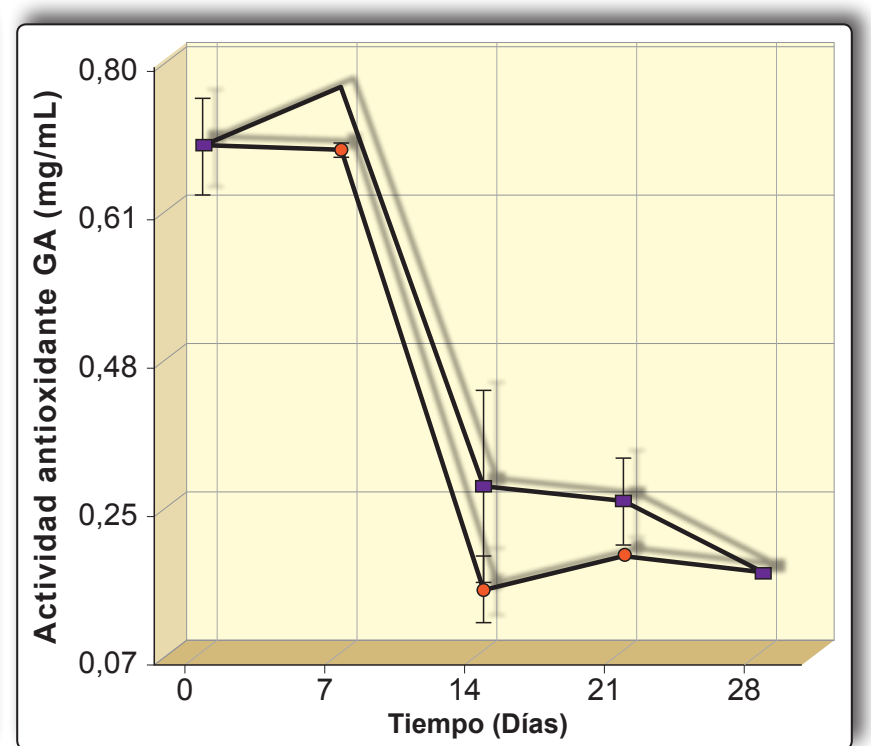

Figura 3. Actividad antioxidante de los formulados de maíz morado y estevia bajo condiciones de temperatura ambiente "๑" y refrigeración " $\square$ ". Valores promedio de 3 repeticiones con su respectiva desviación estándar.

\section{Prueba del grado de satisfacción}

Se trabajó con una muestra no probabilística constituida por 70 asistentes al Instituto de Cultura Alimentaria-INCAA de la ciudad de Lima. Todos los participantes cumplieron los criterios de inclusión: (a) ser participantes activos del INCAA, (b) tener conocimiento de salud alimentaria, (c) aceptar ser partícipe de la investigación.

El Instrumento utilizado para la realizar la prueba se construyó según escala hedónica de 9 categorías: (9) me gusta extremadamente, (8) me gusta mucho, (7) me gusta bastante, (6) me gusta ligeramente, (5) ni me gusta ni me disgusta, (4) me disgusta ligeramente, (3) me disgusta bastante, (2) me disgusta mucho, y (1) me disgusta extremadamente; sobre los atributos de color, olor, sabor y aceptabilidad general del formulado ${ }^{(18-20)}$.

\section{Análisis de datos}

Todos los resultados se procesaron en el programa Excel para Windows 2007.

\section{RESULTADOS Y DISCUSIÓN}

\section{Composición del formulado}

Los resultados del análisis cromatográfico (tabla 1) en el formulado muestran compuestos tales como: derivado del ácido protocatéquico $(136,17 \mathrm{mg} / 100$ $\mathrm{mL}$ ); derivados de antocianina $26,40 \mathrm{mg} / 100 \mathrm{~mL} ; 14,18$ $\mathrm{mg} / 100 \mathrm{~mL} ; 6,42 \mathrm{mg} / 100 \mathrm{~mL}$ y $2,42 \mathrm{mg} / 100 \mathrm{~mL}$; y los derivados del ácido cinámico $(2,29 \mathrm{mg} / 100 \mathrm{~mL}$ y 1,99 


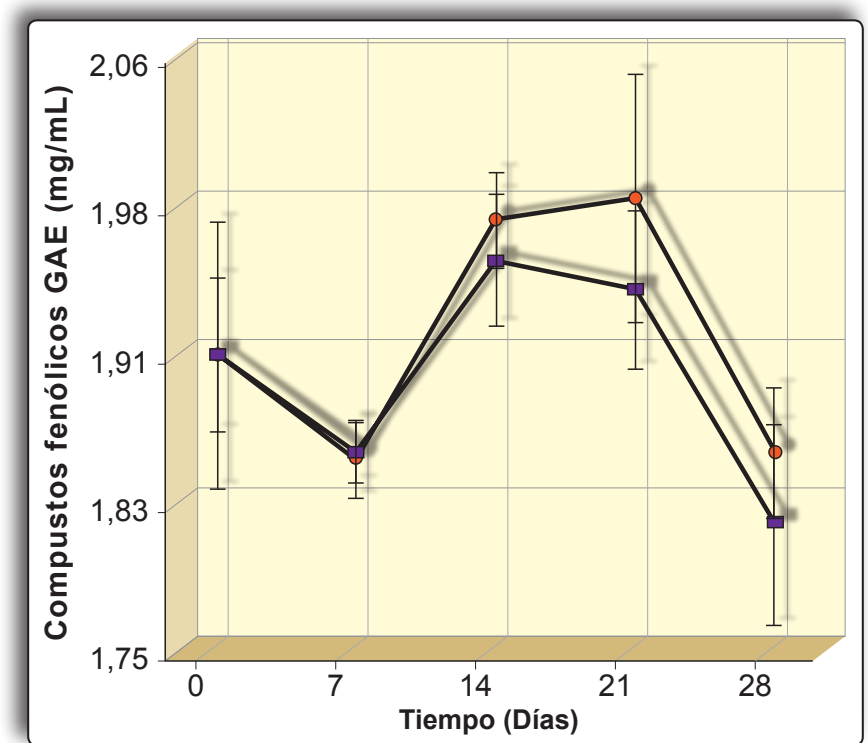

Figura 4. Compuestos fenólicos de los formulados de maíz morado y estevia bajo condiciones de temperatura ambiente "๑" y refrigeración "ם". Valores promedio de 3 repeticiones con su respectiva desviación estándar.

$\mathrm{mg} / 100 \mathrm{~mL}$ ). El contenido total de estos compuestos fenólicos según los resultados hallados fue de 200,09 $\mathrm{mg} / 100 \mathrm{~mL}$, siendo similar al encontrado en la determinación de compuestos fenólicos utilizando el método de Folin-Ciocalteu. En cuanto al contenido de antocianinas, el valor total determinado por HPLC fue de 50,84 mg/100 mL, que se encontró por encima de los valores hallados por el método espectrofotométrico. Otros investigadores, en el estudio del perfil de fenólicos de las corontas del maíz morado a través de HPLC-PAD, encontraron en un extracto atomizado comercial de origen peruano valores en su fracción acuosa (FA) ${ }^{(21)}$.

\section{Actividad antioxidante del formulado}

La capacidad antioxidante del formulado según el método ABTS fue 17,96 $\mu$ mol de equivalentes Trolox/ $\mathrm{mL}$; 31,o1 $\mu \mathrm{mol}$ de equivalentes Trolox/mL utilizando el método ORAC y o,706 equivalentes de ácido gálico $\mathrm{mg} / \mathrm{mL}$ según el método DPPH.

Al respecto, la investigación realizada en Italia sobre la capacidad antioxidante total en hortalizas, frutos, bebidas libres de alcohol, bebidas alcohólicas, tés, cafés y aceites consumidos en ese país por los métodos FRAP, TRAP y ABTS, cuyos resultados se expresaron como equivalentes Trolox (TEAC), siendo en todos los casos inferiores a los encontrados en la investigación para el formulado de maíz morado y estevia $(17,96 \mathrm{mmol} / \mathrm{L})$ ${ }^{(22)}$. De acuerdo a los valores de ABTS en equivalentes TEAC el formulado presenta una significativa actividad antioxidante que puede muy bien sustituir bebidas

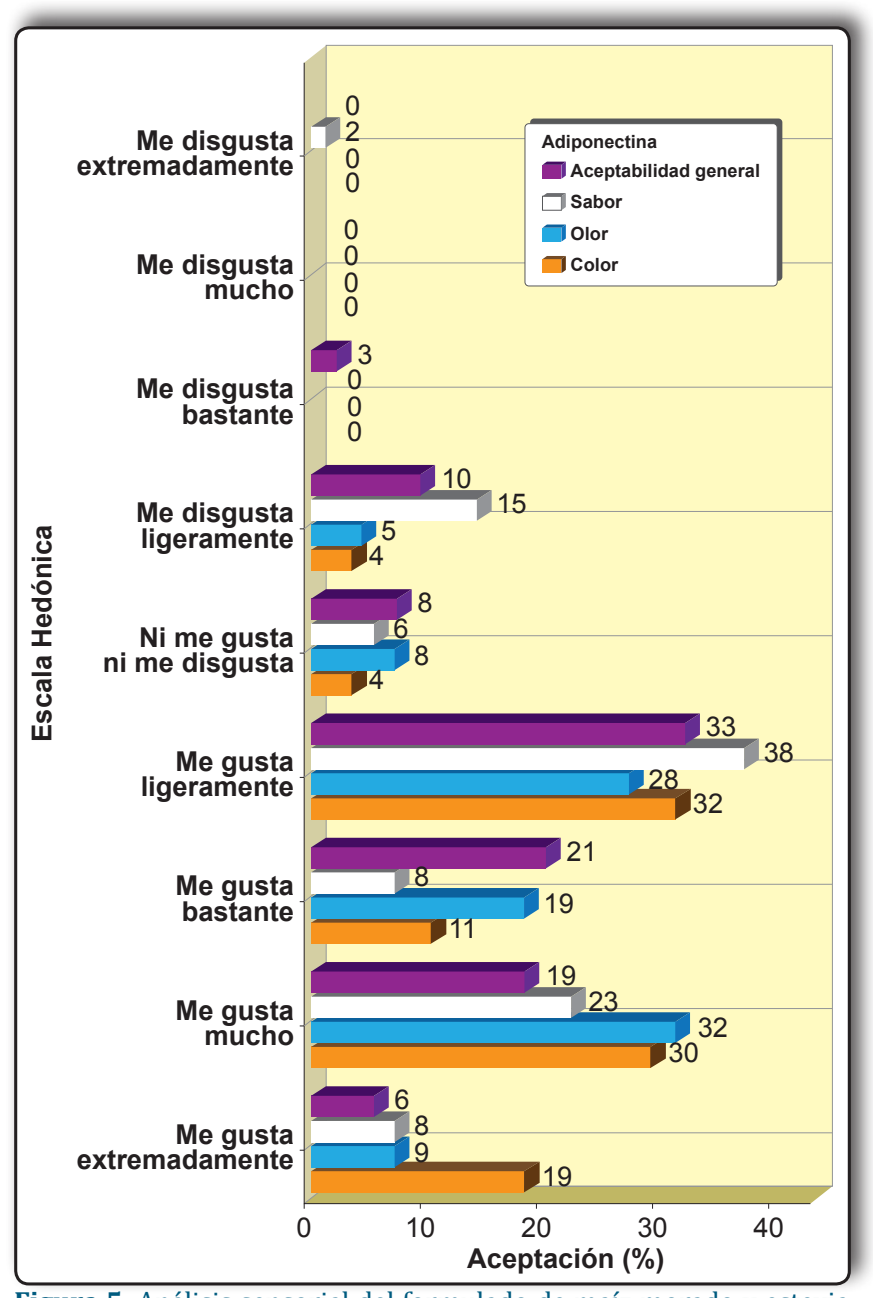

Figura 5. Análisis sensorial del formulado de maíz morado y estevia sobre los atributos de aceptabilidad en general, color, olor y sabor.

comerciales carbonatadas ricas en fructosa, sacarosa y colorantes que producen riesgos sobre la salud humana ${ }^{(23)}$. Sin embargo, con respecto a otros tipos de bebidas como los cafés expreso, expreso y descafeinado, extracto y soluble, los valores en TEAC fueron en todos los casos superiores al formulado de maíz morado y estevia $(26,96$ $-36,54 \mathrm{mmol}$ TEAC/L) ${ }^{(22)}$.

\section{Estabilidad de antocianinas en el formulado}

La estabilidad de los formulados se evaluó bajo condiciones de temperatura ambiente y refrigeración, a $\operatorname{los} 7,14,21$ y 28 días. La evaluación indica que las antocianinas a temperatura ambiente y refrigeración $\left(5-8^{\circ} \mathrm{C}\right)$ presentaron valores entre 0,20 y $0,28 \mathrm{mg} / \mathrm{mL}$, y que estas fueron superiores para los conservados bajo refrigeración, para los 7, 14 y 21 días (figura 2); sin embargo, para los 28 días los valores fueron similares, y se explican por el posible efecto sinérgico entre los componentes presentes en el formulado. 
El estudio realizado por Chun-Ying et al. ${ }^{(24)}$, relacionadocon el efecto antioxidantedel extracto de maíz morado sobre la mayonesa almacenada por 10 semanas, reveló que las antocianinas son poderosos antioxidantes cuyo efecto sobre la estabilidad de los lípidos de la mayonesa en términos de valor de peróxido, p-anisidina, oxidación total, valor ácido y valor de iodo durante ese tiempo resultó ser mejor que los antioxidantes químicos BHT y EDTA. En ese sentido, los resultados observados por estos investigadores se encuentran de acuerdo con la estabilidad relativa que muestran las antocianinas dentro del formulado; de acuerdo a estos investigadores y los resultados hallados en este estudio, las antocianinas del maíz morado se pueden utilizar como sustitutos de antioxidantes químicos en la industria alimentaria.

Estabilidad de la actividad antioxidante en el formulado

En el caso de la actividad antioxidante expresada como equivalentes de ácido gálico $(\mathrm{mg} / \mathrm{mL})$ según el método DPPH.

Los resultados muestran una disminución de la actividad antioxidante con el tiempo de almacenamiento bajo condiciones de refrigeración y temperatura ambiente hasta los 14 días. Después de este tiempo se observa una relativa estabilidad de este marcador alrededor de 0,22 mg GAE/mL de bebida (figura 3), disminución que se explica por la inactividad del ácido ascórbico que se agregó a la bebida durante su acondicionamiento.

\section{Estabilidad de los compuestos fenólicos en el formulado}

Los compuestos fenólicos en el formulado se encontraron entre 1,83 a $2 \mathrm{mg} \mathrm{GAE} / \mathrm{mL}$ de bebida para los frascos mantenidos a temperatura ambiente y bajo refrigeración (figura 4). De acuerdo a los resultados, los compuestos fenólicos mostraron una relativa estabilidad durante 28 días, lo que revela que los compuestos de naturaleza fenólica presentesen esteviay maíz moradoson relativamente estables, concordando con investigaciones realizadas sobre la estabilidad de fenólicos aplicados a mayonesas y con valores superiores a los presentados por las infusiones de té verde chino y té negro ${ }^{(24,25)}$.

\section{Evaluación dela prueba del grado desatisfacción}

La evaluación sensorial para el formulado de maíz morado y estevia se realizó en una población de 70 personas, de las cuales el $67 \%$ fueron mujeres y el $33 \%$ hombres. En cuanto a los rangos de edades, el 56\% de los encuestados fueron mayores de 50 años, el $28 \%$ estuvo entre los 40 y 50 años, y el $17 \%$ restante entre los
20 y 30 años. Los resultados sobre la escala hedónica referente al grado de satisfacción, teniendo en cuenta los atributos de aceptabilidad en general, color, olor y sabor se muestran en la figura 5 .

El resultado de la prueba muestra que el formulado se califica principalmente entre "me gusta ligeramente" (33\%) y "me gusta mucho" (19\%) según la escala hedónica ${ }^{(18,19)}$. Al respecto no se encuentran antecedentes para el formulado de maíz morado y estevia y consideramos que estos resultados motivan emprendimientos para el desarrollo de otros tipos de preparaciones con el maíz morado y estevia.

\section{CONCLUSIONES}

El formulado de maíz morado y estevia presenta alrededor de $2 \mathrm{mg} / \mathrm{mL}$ en compuestos fenólicos, alto contenido de antocianinas $(50,84 \mathrm{mg} / 100 \mathrm{~mL}$ de formulado) según HPLC-PAD y sobresaliente actividad antioxidante según ABTS $(17,96 \mu \mathrm{mol} \mathrm{ET} / \mathrm{mL})$, ORAC (31,01 $\mu \mathrm{mol} \mathrm{ET/mL)} \mathrm{y} \mathrm{DPPH}(0,706 \mathrm{GAE} \mathrm{mg} / \mathrm{mL})$, por lo que es una bebida funcional. El grado de satisfacción califica a la bebida entre "me gusta mucho" (19\%) y "me gusta ligeramente" (33\%) según escala hedónica sobre los atributos de aceptabilidad general, sabor, olor y color.

AGRADECIMIENTO: Los autores agradecemos al CONCYTEC por el financiamiento al proyecto de investigación.

\section{REFERENCIAS BIBLIOGRÁFICAS}

1. Cárdenas H, Sánchez J, Roldan L, Mendoza F. Prevalencia del síndrome metabólico en personas a partir de 20 años de edad. Perú, 2005. Rev Esp Salud Pública. 2009; 83 (2): 257-65.

2. Vásquez-Kunze S, Málaga G. Las nuevas guías de hipertensión arterial y dislipidemia, más allá de la controversia, ¿son guías confiables? Rev Peru Med Exp Salud Pública. 2014; 31(1): 143-50.

3. World Health Organization. Global status report on Noncommunicable Diseases, 2010. [Internet]. [Citado 03 marzo 2013]. Disponible en: http://www.who.int/nmh/ publications/ncd_report_full_en.pdf

4. Vinueza R, Boissonnet CP, Acevedo M, Uriza F, Benitez FJ, Silva H, et al. Dyslipidemia in seven Latin American cities: CARMELA study. Prev Med. 2010; 50(3): 106-11.

5. Ronceros G, Ramos W, Arroyo J, Galarza C, Gutiérrez E, Ortega-Loayza A, et al. Estudio comparativo del maíz morado (Zea mays L.) y simvastatina en la reducción de lípidos séricos de pacientes diabéticos normotensos con dislipidemia. An Fac Med. 2012; 73(2): 113-17.

6. Tsuda T, Horio F, Uchida K, Aoki H, Osawa T. Dietary cyaniding 3-O-beta-D-glucoside-rich purple corn color 
prevents obesity and ameliorates hyperglycemia in mice. J Nutr. 2003; 133(7): 2125-30.

7. Kwon SH, Ahn IS, Kim SO, Kong CS, Chung HY, Do MS, et al. Anti-obesity and hypolipidemic effects of black soybean anthocyanins. J Med Food. 2007; 10(3): 552-6.

8. Arroyo J, Raez E, Rodríguez M, Chumpitaz V, Burga J, De la Cruz W, et al. Reducción del colesterol y aumento de la capacidad antioxidante por el consumo crónico de maíz morado (Zea mays L) en ratas hipercolesterolémicas. Rev Peru Med Exp Salud Pública. 2007; 24(2): 157-62.

9. Arroyo J, Raez E, Rodríguez M, Chumpitaz V, Burga J, De la Cruz W, et al. Actividad antihipertensiva y antioxidante del extracto hidroalcohólico atomizado de Maíz morado (Zea mays L) en ratas. Rev Peru Med Exp Salud Pública. 2008; 25(2): 195-9.

10. Kim JY, Hong JH, Jung HK, Jeong YS, Cho KH. Grape skin and loquat leaf extracts and acai puree have potent antiatherosclerotic and anti-diabetic activity in vitro and in vivo in hypercholesterolemic zebrafish. International Journal of Molecular Medicine. 2012; 30 (3): 606-14.

11. Geeraert B, Crombé $F$, Hulsmans $M$, Benhabilès $N$, Geuns J, Holvoet P. Stevioside inhibits atherosclerosis by improving insulin signalling and antioxidant defence in obese insulin-resistant mice. Inter J Obesity. 2010, 34 (3); 569-77.

12. Giusti MM, Wrosltad RE. Characterization and measurements of anthocyanins by UV-VIS spectroscopy. En: Current protocols in food analytical chemistry. New York: John Wiley \& sons. Inc.; 2001. p. 13.

13. Quispe F, Arroyo K, Gorriti A. Características morfológicas y químicas de 3 cultivares de maíz morado (Zea mays L.) en Arequipa-Perú. Rev Soc Quím Perú. 2011; 77(3): 205-17.

14. Rivero A, Betancort J. Evaluación de la Actividad Antioxidante de Polifenoles de Algas Marinas. [Práctica de Laboratorio (Práctica VI.3)]. [Internet]. España: Universidad de Las Palmas de Gran Canaria; 20o6. , 2006: 3. [Citado 13 octubre 2009]. Disponible en: http:// old.iupac.org/publications/cd/medicinal_chemistry/ Practica-VI-3.pdf

15. Arnao M. Some methodological problems in the determination of antioxidant activity using chromogen radicals: a practical case. Trends in Food Science \& Technology. 2000; 11(11): 419-21.

16. Huang D, Ou B, Hampsch-Woodill, MF, Judith A, Prior RL. High throughput assay of oxygen radical absorbance capacity (ORAC) using a multichannel liquid handling system coupled with a microplate fluorescence reader in 96-well format. Journal of Agricultural and Food Chemistry. 2002; 50(16): 4437-44.

17. Chirinos R, Campos D, Costa N, Arbizu C, Pedreschi $\mathrm{R}$, Larondelle Y. Phenolic profiles of Andean mashua (Tropaeolum tuberosum Ruiz \& Pavón) tubers: Identification by HPLC-DAD and evaluation of their antioxidant activity. Food Chemistry. 2008; 106(3): 1285-98.

18. Schnettler B, Miranda H, Sepúlveda J, Denegri M. Satisfacción con la alimentación y la vida, un estudio exploratorio en estudiantes de la frontera, Temuco-Chile. Psicologia \& Sociedade. 2011; 23(2): 426-35.

19. Basuki A, Ikhsani T, Suryatman S. Physical, sensorial and chemical characteristics of simulated chips of cassava (Manihot esculenta Crantz): Rice (Oryza sativa L.) mix. Procedia Food Science. 2015; 3: 82-95.

20. Pilgrim FJ, Schutz HG, Peryam DR. Influence of monosodium glutamate on taste perception. Journal of Food Science. 1955; 20 (4): 310-4.

21. Pedreschi P, Cisneros-Zevallos L. Pheonolic profiles of Andean purple corn (Zea mays L.). Food Chemistry. 2007; 100 (3): 956-63.

22. Pellegrini N, Serafini M, Colombi B, Del Rio D, Salvatore $\mathrm{S}$, Bianchi $\mathrm{M}$, et al. Total antioxidant capacity of plant food, beverages and oils consumed in Italy assessed by three different in vitro assays. J. Nutr. 2003; 133(9): 2812-9.

23. Yu B, He H, Zhang Q, Wu H, Du H, Liu L, et al. Soft drink consumption is associated with depressive symptoms among adults in China. Journal of Affective Disorders. 2014; 172C: $422-7$.

24. Li CY, Kim HW, Li H, Lee DC, Rhee HI. Antioxidant effect of purple corn extracts during storage of mayonnaise. Food Chemistry. 2014; 152: 592- 6.

25. Deetae P, Parichanon P, Trakunleewatthana P, Chanseetis Ch, Lertsiri S. Antioxidant and anti-glycation properties of Thai herbal teas in comparison with conventional teas. Food Chemistry 2012; 133(3): 953-9.

Manuscrito recibido el: 06/04/15

Aceptado para su publicación el: 20/05/15

\section{Correspondencia:}

Nombre: $\quad$ Pablo Enrique Bonilla Rivera

Dirección: Jr. Puno 1002-FFB-UNMSM Lima-Perú. E-mail:_pbonillar@unmsm.edu.pe 